\title{
Blind Selected Mapping with Side Information Estimation Based on the Received Pilot Signal
}

\author{
Eunchul Yoon $\mathbb{D}^{1},{ }^{1}$ Duckdong Hwang, ${ }^{2}$ Chanki Jang $\mathbb{D}^{1},{ }^{1}$ Janghyun Kim, $^{1}$ and Unil Yun $\mathbb{D}^{3}$ \\ ${ }^{1}$ Department of Electrical and Electronics Engineering, Konkuk University, Seoul, Republic of Korea \\ ${ }^{2}$ Department of Electronics, Information and Communication Engineering, Sejong University, Seoul, Republic of Korea \\ ${ }^{3}$ Department of Computer Engineering, Sejong University, Seoul, Republic of Korea \\ Correspondence should be addressed to Unil Yun; yunei@sejong.ac.kr
}

Received 25 December 2017; Accepted 8 May 2018; Published 28 June 2018

Academic Editor: Ernestina Cianca

Copyright (C) 2018 Eunchul Yoon et al. This is an open access article distributed under the Creative Commons Attribution License, which permits unrestricted use, distribution, and reproduction in any medium, provided the original work is properly cited.

\begin{abstract}
Blind selected mapping (BSLM) is a technique to reduce the peak to average power ratio (PAPR) of OFDM by using a set of scrambling sequences. Since the side information (SI) on the scrambling sequence selected by the transmitter is not directly sent to the receiver in BSLM, the SI needs to be estimated by the receiver. In this paper, a BSLM scheme that applies a new SI estimation approach is proposed. The proposed BSLM scheme estimates the SI by selecting the minimum of the candidate metrics that are computed with the received pilot signal, the pilot symbol sequence, and the scrambling sequences. It is shown by simulation that the proposed BSLM scheme yields better SI error rate (SIER) and bit error rate (BER) performance and lower implementation complexity than four traditional BSLM schemes.
\end{abstract}

\section{Introduction}

Orthogonal frequency division multiplexing (OFDM) is an attractive transmission technique for broadband communication. OFDM can overcome the detrimental impact of intersymbol interference efficiently without requiring complex equalization [1]. Since OFDM is considered as appropriate for high spectral efficiency and scalable to low complexity receivers, it is well suited for the 5G cellular network [2]. However, OFDM has a critical problem of high peak to average power ratio (PAPR). When the signal with high PAPR passes nonlinear devices such as digital-to-analog converter and high power amplifier, the problems of quantization noise, in-band signal distortion, and out-of-band radiation can be severely aggravated $[3,4]$. Therefore, various PAPR reduction schemes have been proposed (e.g., [517]). Selected mapping (SLM) introduced in [18] has been known as an efficient PAPR reduction technique with low implementation complexity. SLM generates multiple candidate transmission sequences by multiplying the data symbol sequence by multiple scrambling sequences and selects a candidate transmission sequence that can lead to the lowest PAPR. To recover the data symbol sequence, the receiver needs the side information (SI) on the scrambling sequence selected by the transmitter. Since correct delivery of the SI is particularly crucial for reliable recovery of the data symbol sequence, the SI should be protected with strong channel coding, which decreases the effective data transfer rate. In order to apply the SLM technique without directly transmitting the SI from the transmitter to the receiver, several blind SLM (BSLM) schemes have been studied. In [19], the maximum likelihood (ML) detection was introduced for BSLM and a simplified ML detection approach was suggested to reduce the complexity of the ML detection. Although the simplified ML detection substantially reduced the complexity, it still had high complexity. In [20], the SI was embedded in the scrambling sequence by partitioning each scrambling sequence into multiple subsequences and multiplying those subsequences by a distinct set of phaseoffsets. While the BSLM scheme in [20] had low complexity, it showed slightly worse bit error rate (BER) performance than other BSLM schemes at a low signal to noise ratio (SNR). Reference [21] proposed a BSLM scheme that embedded the SI in the scrambling sequence by allocating power-extended scrambling symbols. While it considerably reduced complexity, its BER performance was deteriorated with a large symbol 
constellation size. Reference [22] proposed a BSLM scheme that estimated the SI by assuming high autocorrelation between the channel frequency response (CFR) coefficients over the adjacent pilot subcarriers. The BSLM scheme in [22] showed low complexity and performed comparatively well even with a large symbol constellation size. However, our simulation results showed that, given a multipath fading channel consisting of a large number of effective channel taps with nontrivial tap powers, the BER performance degraded severely. It is because a greater number of channel taps can cause more fluctuations in the CFR amplitudes leading to more weakened autocorrelation between the CFR coefficients over the adjacent pilot subcarriers. Reference [23] proposed a BSLM scheme to decrease the number of the subcarriers that were used to indirectly help to estimate the SI. However, at the expense of reducing the number of subcarriers, the BER performance of the BSLM scheme became deteriorated.

In this paper, a BSLM scheme that applies a new SI estimation approach is proposed. The proposed BSLM scheme estimates the SI by selecting the minimum of the candidate metrics that are computed with the received pilot signal, the pilot symbol sequence, and the scrambling sequences. It is shown by simulation that the proposed BSLM scheme yields better SI error rate (SIER) and BER performance and lower implementation complexity than four traditional BSLM schemes.

The remainder of this paper is organized as follows. In Section 2, the system model is explained. In Section 3, the previous BSLM schemes are introduced. In Section 4, the proposed BSLM scheme is explained and its complexity is analyzed. In Section 5, the SIER and BER performances of the proposed BSLM scheme and its implementation complexity are compared with those of the conventional BSLM schemes. Finally, in Section 6, this paper is concluded.

Notations. $(\cdot)^{T}$ and $(\cdot)^{H}$ denote the transpose operator and the complex conjugate transpose operator, respectively. $\mathbb{U}(a, b)$ denotes the uniform distribution with its random variable located between $a$ and $b$. $\|\cdot\|$ denotes the Frobenius norm operator. $\operatorname{diag}\{\mathbf{X}\}$ denotes a diagonal matrix whose diagonal components are given by the components of a vector $\mathbf{X}$. $\mathbf{D}(m, n)$ denotes the component of a matrix $\mathbf{D}$ in the $m$-th row and the $n$-th column. Finally, $\mathbf{I}_{N}$ denotes an $N \times N$ identity matrix.

\section{System Model}

We consider an OFDM system with a block size $K=K_{d}+K_{p}$, where $K_{d}$ and $K_{p}$ denote the number of data subcarriers and the number of pilot subcarriers, respectively. The channel impulse response is modeled by a finite impulse response filter with maximum length $L$ as

$$
\mathbf{h}=\left[\begin{array}{llll}
h_{0} & h_{1} & \cdots & h_{L-1}
\end{array}\right]^{T},
$$

where $\left\{h_{l}\right\}_{l=0}^{L-1}$ are uncorrelated complex Gaussian random variables with zero mean. The average power of $h_{l}$ for $l=0,1, \cdots, L-1$ is given by the exponential power delay profile (PDP) [24, 25]:

$$
E\left\{\left|h_{l}\right|^{2}\right\}=\gamma e^{-l\left(T_{S} / \sigma_{T}\right)}
$$

where $\gamma$ denotes a power normalization factor that guarantees $E\left\{\sum_{l=0}^{L-1}\left|h_{l}\right|^{2}\right\}=1$ and $T_{S}$ denotes the sampling period with $1 / T_{S}$ being the system bandwidth, and $\sigma_{T}$ denotes the root mean square channel delay spread. With a large valued $\sigma_{T} / T_{S}$, the average channel tap power decays slowly with increasing $l$, which means there are a larger number of effective channel taps with nontrivial tap powers. The CFR coefficient vector,

$$
\mathbf{H}=\left[\begin{array}{llll}
H_{0} & H_{1} & \cdots & H_{K-1}
\end{array}\right]^{T},
$$

is given by the $K$-point discrete Fourier transform of the channel impulse response. If a $K \times L$ matrix $\mathbf{D}$ is defined by

$$
\mathbf{D}(k, l)=e^{(-j 2 \pi / K) k l},
$$

for $k=0,1, \cdots, K-1$ and $l=0,1, \cdots, L-1$, the CFR coefficient vector $\mathbf{H}$ is given by

$$
\mathbf{H}=\mathbf{D h} \text {. }
$$

The data symbols are chosen from an $M$-ary QAM constellation, $Q$, which has unit average symbol power, and the pilot symbols are chosen from the BPSK constellation, $\{-1,+1\}$. Data and pilot symbols are mapped to an OFDM block to form an OFDM symbol sequence:

$$
\mathbf{X}=\left[X_{0}, X_{1}, \cdots, X_{K-1}\right]^{T} .
$$

With the pilot subcarrier indices given by $p_{0}, p_{1}, \cdots$, and $p_{K_{p}-1}$, the pilot symbol sequence can be written as

$$
\mathbf{X}_{p}=\left[X_{p_{0}}, X_{p_{1}}, \cdots, X_{p_{K_{p}-1}}\right]^{T}
$$

and the CFR coefficient vector for the pilot subcarriers can be written as

$$
\mathbf{H}_{p}=\left[H_{p_{0}}, H_{p_{1}}, \cdots, H_{p_{K_{p}-1}}\right]^{T}
$$

With the data subcarrier indices given by $d_{0}, d_{1}, \cdots$, and $\left.d_{K_{d}-1}\right\}$, the data symbol sequence $\mathbf{X}_{d}$ and the CFR coefficient vector for the data subcarriers $\mathbf{H}_{d}$ are defined analogously. Note that $\mathbf{H}_{p}$ can be written in terms of $\mathbf{h}$ as

$$
\mathbf{H}_{p}=\mathbf{D}_{p} \mathbf{h},
$$

where $\mathbf{D}_{p}$ is an $K_{p} \times L$ matrix given by

$$
\mathbf{D}_{p}(k, l)=e^{(-j 2 \pi / K) k l},
$$

for $k=p_{0}, p_{1}, \cdots, p_{K_{p}-1}$ and $l=0,1, \cdots, L-1$. The timedomain sample sequence,

$$
\mathbf{x}=\left[x_{0}, x_{1}, \cdots, x_{K-1}\right]^{T},
$$

is given by the inverse discrete Fourier transform (IDFT) of $\mathbf{X}$. The PAPR of OFDM is defined by

$$
\operatorname{PAPR}=\frac{\max _{0 \leq n \leq K-1}\left|x_{n}\right|^{2}}{(1 / K) \sum_{n=0}^{K-1}\left|x_{n}\right|^{2}} .
$$


In SLM, the transmitter generates $U$ scrambled sequences in frequency domain by multiplying $\mathbf{X}$ by $U$ scrambling sequences:

$$
\boldsymbol{\Phi}^{(u)}=\left[e^{j \phi_{0}^{(u)}}, e^{j \phi_{1}^{(u)}}, \cdots, e^{j \phi_{K-1}^{(u)}}\right]^{T}
$$

for $u=0,1, \cdots, U-1$. The scrambling sequences $\left\{\Phi^{(u)}\right\}_{u=0}^{U-1}$ are assumed to be known to both the transmitter and the receiver. The transmitter generates $U$ candidate timedomain sample sequences by taking the IDFT of each scrambled sequence and selects a candidate time-domain sample sequence that can lead to the minimum PAPR. In BSLM, the receiver estimates the SI, i.e., the index of the scrambling sequence selected by the transmitter. If the SI is $\widetilde{u}$, the received signal over the $k$-th subcarrier for $k=0,1, \cdots, K-1$ can be written as

$$
Y_{k}=H_{k} e^{j \phi_{k}^{(\tilde{u})}} X_{k}+W_{k}
$$

where $W_{k}$ denotes the additive Gaussian noise with mean zero and variance $\sigma^{2}$.

\section{Previous BSLM Schemes}

In this section, traditional BSLM schemes are described for comparison purposes.

3.1. The Conventional ML-Based BSLM Scheme. The conventional ML-based BSLM scheme can be written as

$$
\begin{aligned}
& \left(\widehat{u}, \widehat{X}_{d_{0}}, \cdots, \widehat{X}_{d_{K_{d}-1}}\right) \\
& =\arg \min _{\substack{u \in\{0,1, \cdots, U-1\} \\
X_{d_{0}} \in Q, \cdots, X_{d_{K^{-1}}} \in Q}} \sum_{k=0}^{K_{d}-1}\left|Y_{d_{k}}-\widehat{H}_{d_{k}} X_{d_{k}} e^{j \phi_{k}^{(u)}}\right|^{2},
\end{aligned}
$$

where $\widehat{H}_{d_{k}}$ denotes the CFR coefficient over the $k$-th data subcarrier estimated at the receiver. In the above, $\phi_{k}^{(u)}$ was defined by $\phi_{k}^{(u)} \sim \mathbb{U}(0,2 \pi)$, which satisfied the condition of $X_{d_{k}} e^{j \phi_{k}^{(u)}} \notin Q$ Q. This scheme requires $U q^{K_{d}}$ computations of the ML metric $\left|Y_{d_{k}}-\widehat{H}_{d_{k}} X_{d_{k}} e^{j \phi_{k}^{(u)}}\right|^{2}$.

3.2. The Simplified ML-Based BSLM Scheme. Reference [19] suggested a simplified ML-based BSLM scheme. The scheme can be summarized as

$$
\widehat{u}=\arg \min _{u \in\{0,1, \cdots, U-1\}} \mathscr{M}^{(u)}
$$

where

$$
\mathscr{M}^{(u)}=\sum_{k=0}^{K_{d}-1} \min _{X_{d_{k}} \in \mathbb{Q}}\left|Y_{d_{k}}-\widehat{H}_{d_{k}} X_{d_{k}} e^{j \phi_{k}^{(u)}}\right|^{2} .
$$

In [19], $\phi_{k}^{(u)}$ was defined by $\phi_{k}^{(u)} \sim \mathbb{U}(0,2 \pi)$, which satisfied the condition of $X_{d_{k}} e^{j \phi_{k}^{(u)}} \notin Q$. In order to reduce implementation complexity, all the values of $\left\{\mathscr{M}^{(u)}\right\}$ can be computed and stored in memory to be used in search of $\widehat{u}$. Once the optimal $\widehat{u}$ is determined in (16), $\mathbf{X}_{d}$ can be estimated by taking the optimal arguments $\left\{\widehat{X}_{d_{k}}\right\}$ of the minimization problems in (17) in search of $\mathscr{M}^{(\hat{u})}$. This scheme requires $U q N_{\text {DATA }}$ computations of the ML metric $\left|Y_{d_{k}}-\widehat{H}_{d_{k}} X_{d_{k}} e^{j \phi_{k}^{(u)}}\right|^{2}$.

3.3. The BSLM Scheme Using Phase Offset Factors. Reference [20] proposed an BSLM scheme that partitioned the scrambling sequence $\mathbf{P}^{(u)}$ into $I$ subsequences and multiplied each subsequence by a phase offset factor to enable the receiver to estimate $\widetilde{u}$ effectively. In [20], $\phi_{k}^{(u)}$ was defined by $\phi_{k}^{(u)} \in$ $\{0, \pi / 2, \pi, 3 \pi / 2\}$, which satisfied the condition of $X_{d_{k}} e^{j \phi_{k}^{(u)}} \epsilon$ Q. The phase offset sequences were given by

$$
\mathbf{C}^{(u)}=\left[c_{0}^{(u)}, c_{1}^{(u)}, \ldots, c_{I-1}^{(u)}\right], \quad c_{i}^{(u)} \in\{0,1\},
$$

for $u=0,1, \cdots, U-1$, which were designed to have the largest minimum Hamming distance. Since the $i$-th scrambling subsequence is multiplied by the phase offset factor $e^{j(\pi / 4) c_{i}^{(u)}}$, the received signal over the $k$-th data subcarrier for $k=$ $0, \cdots, K_{d}-1$, can be written as

$$
Y_{d_{k}}=H_{d_{k}} X_{d_{k}} e^{j \phi_{k}^{(\tilde{u})}} e^{j(\pi / 4) c_{i}^{(\tilde{u})}}+N_{d_{k}}
$$

where $i=\left\lfloor k I / K_{d}\right\rfloor$. With the definition of $S_{k}=X_{d_{k}} e^{j \phi_{k}^{(\tilde{u})}}(\epsilon$ Q), the BSLM scheme in [20] can be summarized as

$$
\widehat{u}=\arg \min _{u \in\{0,1, \cdots, U-1\}} \sum_{i=0}^{I-1} \mathscr{M}^{\left(i, c_{i}^{(u)}\right)},
$$

where

$$
\mathscr{M}^{(i, c)}=\sum_{k=i K_{d} / I}^{(i+1) K_{d} / I-1} \min _{S_{k} \in \mathscr{Q}}\left|Y_{d_{k}}-\widehat{H}_{d_{k}} S_{k} e^{j(\pi / 4) c}\right|^{2},
$$

for $c=0,1$. In order to reduce implementation complexity, all the values of $\left\{\mathscr{M}^{(i, c)}\right\}$ can be computed and stored in memory to be used in search of $\widehat{u}$. Once the optimal $\widehat{u}$ is determined in (20), $\mathbf{X}_{d}$ can be estimated by taking the optimal arguments $\left\{\widehat{S}_{k}\right\}$ of the minimization problems in (21) in search of $\mathscr{M}^{\left(i, c_{i}^{(\hat{u})}\right)}$ with $i=\left\lfloor k I / K_{d}\right\rfloor$, and then by converting $\left\{\widehat{S}_{k}\right\}$ into $\left\{\widehat{X}_{d_{k}}\right\}$ based on $\widehat{X}_{d_{k}}=\widehat{S}_{k} e^{-j \phi_{k}^{(\hat{u})}}$. This scheme requires $2 q N_{\text {DATA }}$ computations of the ML metric $\left|\mathrm{Y}_{d_{k}}-\widehat{H}_{d_{k}} S_{k} e^{j(\pi / 4) c}\right|^{2}$.

3.4. The BSLM Scheme Using CFR Autocorrelation. Reference [22] proposed an BSLM scheme that exploited CFR autocorrelation to estimate $\tilde{u}$ under the assumption of high correlation between two CFR coefficients over two neighboring pilot subcarriers. In [22], the scrambling sequences were defined by

$$
\mathbf{P}^{\prime(u)}=\left[e^{j \phi_{0}^{(u)}}, e^{j \phi_{1}^{(u)}}, \cdots, e^{j \phi_{N-1}^{(u)}}\right]
$$

for $u=0,1, \cdots, U-1$, and used to scramble $\mathbf{X}$ instead of $\mathbf{X}_{d}$. In [22], $\phi_{k}^{(u)}$ was defined by $\phi_{k}^{(u)} \sim \mathbb{U}(0,2 \pi)$, which satisfied 
$X_{k} e^{j \phi_{k}^{(u)}} \notin Q$. The received signal carrying the $m$-th pilot symbol for $m=0, \cdots, K_{p}-1$, can be written as

$$
Y_{p_{m}}=H_{p_{m}} X_{p_{m}} e^{j \phi_{p_{m}}^{(\tilde{u})}}+W_{p_{m}}
$$

The BSLM scheme in [22] can be summarized as

$$
\widehat{u}=\arg \max _{u \in\{0,1, \cdots, U-1\}} \mathscr{M}^{(u)},
$$

where

$$
\begin{aligned}
& \mathscr{M}^{(u)}=\sum_{m=1}^{K_{p}-1} \mathfrak{R}\left\{G_{p_{m}}^{(u)} G_{p_{m-1}}^{(u) *}\right\}, \\
& G_{p_{m}}^{(u)}=Y_{p_{m}} e^{-j \phi_{p_{m}}^{(u)}} X_{p_{m}} .
\end{aligned}
$$

Note that $G_{p_{m}}^{(\widetilde{u})}$ corresponds to the CFR coefficient estimated over the $m$-th pilot subcarrier, whereas $G_{p_{m}}^{(u)}$ with $u \neq \tilde{u}$ is irrespective of the CFR coefficient over the $m$-th pilot subcarrier. If the autocorrelation between two CFR coefficients over two neighboring pilot subcarriers is very high, it follows that $\mathfrak{R}\left\{G_{p_{m}}^{(\widetilde{u})} G_{p_{m-1}}^{(\widetilde{u}) *}\right\} \approx\left|G_{p_{m}}^{(\widetilde{u})}\right|^{2}$. Therefore, it is highly probable that $\mathscr{M}^{(u)}$ in (25) can be maximized with $u=\tilde{u}$. In order to reduce implementation complexity, all the values of $\left\{\mathscr{M}^{(u)}\right\}$ can be computed and stored in memory to be used in search of $\widehat{u}$. Once the optimal $\widehat{u}$ is determined in (24), $\mathbf{X}_{d}$ can be estimated by

$$
\widehat{X}_{d_{k}}=\arg \min _{X_{d_{k}} \in \mathbb{Q}}\left|Y_{d_{k}}-\widehat{H}_{d_{k}} X_{d_{k}} e^{j \phi_{d_{k}}^{(\hat{u})}}\right|^{2}
$$

for $k=0, \cdots, K_{d}-1$. This scheme performs well when correlation between two CFR coefficients over two neighboring pilot subcarriers is high. However, its performance can be seriously degraded with a large number of channel multipaths because the autocorrelation between two CFR coefficients over two neighboring pilot subcarriers decreases with an increasing number of channel multipaths. This phenomenon will be certified by simulation in Section 5 .

\section{Proposed BSLM Scheme}

In the proposed BSLM scheme, $\phi_{k}^{(u)}$ is chosen as $\phi_{k}^{(u)} \sim$ $\mathbb{U}(0,2 \pi)$ under a constraint of $e^{j \phi_{k}^{(u)}} X_{k} \notin \mathbb{Q}$. The scrambling sequence for the pilot symbols is defined as

$$
\boldsymbol{\Phi}_{p}^{(u)}=\left[e^{j \phi_{p_{0}}^{(u)}}, e^{j \phi_{p_{1}}^{(u)}}, \cdots, e^{j \phi_{P_{K-1}}^{(u)}}\right]^{T} .
$$

The received signal on the pilot subcarriers can be written as

$$
\mathbf{Y}_{p}=\operatorname{diag}\left\{\Phi_{p}^{(\widetilde{u})}\right\} \operatorname{diag}\left\{\mathbf{X}_{p}\right\} \mathbf{H}_{p}+\mathbf{W}_{p} \text {, }
$$

where

$$
\mathbf{W}_{p}=\left[W_{p_{0}}, W_{p_{1}}, \cdots, W_{p_{K_{p}-1}}\right]^{T}
$$

In the proposed scheme, the received pilot signal, $\mathbf{Y}_{p}$, is multiplied by $\operatorname{diag}\left\{\mathbf{X}_{p}\right\}$ and then multiplied by every matrix of $\operatorname{diag}\left\{\boldsymbol{\Phi}_{p}^{(u)}\right\}^{H}$ for $u=0,1, \cdots, U-1$ to yield

$$
\mathbf{Z}_{p}^{(u)}=\operatorname{diag}\left\{\boldsymbol{\Phi}_{p}^{(u)}\right\}^{H} \operatorname{diag}\left\{\mathbf{X}_{p}\right\} \mathbf{Y}_{p} .
$$

If $u=\tilde{u}$, the expression in (31) reduces to

$$
\mathbf{Z}_{p}^{(u)}=\mathbf{D}_{p} \mathbf{h}+\mathbf{W}_{p}^{\prime(u)},
$$

where

$$
\mathbf{W}_{p}^{\prime(u)}=\operatorname{diag}\left\{\boldsymbol{\Phi}_{p}^{(u)}\right\}^{H} \operatorname{diag}\left\{\mathbf{X}_{p}\right\} \mathbf{W}_{p} .
$$

We assume that the components of $\mathbf{W}_{p}^{\prime(u)}$ are independent and identically distributed Gaussian random variables with mean zero and variance $\sigma^{2}$. If $K_{p} \geq L$, based on the least square method [26], the channel impulse response can be approximated in terms of $\mathbf{Z}_{p}^{(u)}$ as

$$
\widehat{\mathbf{h}} \approx\left(\mathbf{D}_{p}^{H} \mathbf{D}_{p}\right)^{-1} \mathbf{D}_{p}^{H} \mathbf{Z}_{p}^{(u)} .
$$

From (9) and (34), the estimated CFR coefficient vector for the pilot subcarriers can be written as

$$
\widehat{\mathbf{H}}_{p}=\mathbf{D}_{p}\left(\mathbf{D}_{p}^{H} \mathbf{D}_{p}\right)^{-1} \mathbf{D}_{p}^{H} \mathbf{Z}_{p}^{(u)} .
$$

Disregarding the noise term and regarding $u$ as the right SI $\tilde{u}$, the received pilot signal, $\mathbf{Y}_{p}$, can be approximated in terms of $\widehat{\mathbf{H}}_{p}$ as

$$
\widehat{\mathbf{Y}}_{p}^{(u)}=\operatorname{diag}\left\{\Phi_{p}^{(u)}\right\} \operatorname{diag}\left\{\mathbf{X}_{p}\right\} \widehat{\mathbf{H}}_{p} .
$$

By substituting $\mathbf{Z}_{p}^{(u)}$ in (31) into (35) and substituting $\widehat{\mathbf{H}}_{p}$ in (35) into (36) sequentially, it follows that

$$
\widehat{\mathbf{Y}}_{p}^{(u)}=\operatorname{diag}\left\{\boldsymbol{\Phi}_{p}^{(u)}\right\} \boldsymbol{\Psi}_{p} \operatorname{diag}\left\{\boldsymbol{\Phi}_{p}^{(u)}\right\}^{H} \mathbf{Y}_{p},
$$

where

$$
\Psi_{p}=\operatorname{diag}\left\{\mathbf{X}_{p}\right\} \mathbf{D}_{p}\left(\mathbf{D}_{p}^{H} \mathbf{D}_{p}\right)^{-1} \mathbf{D}_{p}^{H} \operatorname{diag}\left\{\mathbf{X}_{p}\right\} .
$$

The error vector between $\widehat{\mathbf{Y}}_{p}^{(u)}$ in (37) and $\mathbf{Y}_{p}$ in (29) is given by

$$
\begin{aligned}
\widehat{\mathbf{Y}}_{p}^{(u)}- & \mathbf{Y}_{p} \\
= & \left(\operatorname{diag}\left\{\boldsymbol{\Phi}_{p}^{(u)}\right\} \boldsymbol{\Psi}_{p} \operatorname{diag}\left\{\boldsymbol{\Phi}_{p}^{(u)}\right\}^{H}-\mathbf{I}_{K_{p}}\right) \\
& \times \operatorname{diag}\left\{\boldsymbol{\Phi}_{p}^{(\widetilde{u})}\right\} \operatorname{diag}\left\{\mathbf{X}_{p}\right\} \mathbf{H}_{p} \\
& +\left(\operatorname{diag}\left\{\boldsymbol{\Phi}_{p}^{(u)}\right\} \boldsymbol{\Psi}_{p} \operatorname{diag}\left\{\boldsymbol{\Phi}_{p}^{(u)}\right\}^{H}-\mathbf{I}_{K_{p}}\right) \mathbf{W}_{p} .
\end{aligned}
$$

The total error between $\widehat{\mathbf{Y}}_{p}^{(u)}$ and $\mathbf{Y}_{p}$ for $u=0,1, \cdots, U-1$ is defined by

$$
\varepsilon^{(u)}=\left\|\widehat{\mathbf{Y}}_{p}^{(u)}-\mathbf{Y}_{p}\right\|^{2}
$$


Since $\mathbf{D}_{p}\left(\mathbf{D}_{p}^{H} \mathbf{D}_{p}\right)^{-1} \mathbf{D}_{p}^{H} \mathbf{H}_{p}=\mathbf{D}_{p}\left(\mathbf{D}_{p}^{H} \mathbf{D}_{p}\right)^{-1} \mathbf{D}_{p}^{H} \mathbf{D}_{p} \mathbf{h}=\mathbf{H}_{p}$, in the case of $u=\widetilde{u}$, the first term in the right-hand side of (39) reduces to zero. It is easy to show that $\Psi_{p}^{H} \Psi_{p}=\Psi_{p}$. Therefore, in the case of $u=\widetilde{u}, \varepsilon^{(u)}$ is given by

$$
\varepsilon^{(\widetilde{u})}=\mathbf{W}_{p}^{H}\left(\mathbf{I}_{K_{p}}-\operatorname{diag}\left\{\boldsymbol{\Phi}_{p}^{(\widetilde{u})}\right\} \boldsymbol{\Psi}_{p} \operatorname{diag}\left\{\boldsymbol{\Phi}_{p}^{(\widetilde{u})}\right\}^{H}\right) \mathbf{W}_{p} .
$$

The averaged total error with respect to $\mathbf{W}_{p}$ reduces to

$$
E\left\{\varepsilon^{(\widetilde{u})}\right\}=\sigma^{2}\left(K_{p}-L\right) .
$$

In the case of $u \neq \tilde{u}, \widehat{\mathbf{Y}}_{p}^{(u)}$ is given by (37) and the error vector between $\widehat{\mathbf{Y}}_{p}^{(u)}$ and $\mathbf{Y}_{p}$ is given by (39). Then, the averaged total error with respect to $\mathbf{W}_{p}$ reduces to

$$
\begin{aligned}
E\left\{\varepsilon^{(u)}\right\}= & \mathbf{H}_{p}^{H} \operatorname{diag}\left\{\boldsymbol{\Phi}_{p}^{(\widetilde{u})}\right\}^{H} \operatorname{diag}\left\{\boldsymbol{\Phi}_{p}^{(u)}\right\} \\
& \times\left(\mathbf{I}_{K_{p}}-\mathbf{D}_{p}\left(\mathbf{D}_{p}^{H} \mathbf{D}_{p}\right)^{-1} \mathbf{D}_{p}^{H}\right) \\
& \times \operatorname{diag}\left\{\boldsymbol{\Phi}_{p}^{(u)}\right\}^{H} \operatorname{diag}\left\{\boldsymbol{\Phi}_{p}^{(\widetilde{u})}\right\} \mathbf{H}_{p} \\
& +\sigma^{2}\left(K_{p}-L\right) .
\end{aligned}
$$

Note that $E\left\{\varepsilon^{(u)}\right\} \geq E\left\{\varepsilon^{(\widetilde{u})}\right\}$ because $E\left\{\varepsilon^{(u)}\right\}-E\left\{\varepsilon^{(\widetilde{u})}\right\}$ can be written in a positive semidefinite matrix structure. Since $\varepsilon^{(u)} \geq 0, \varepsilon^{(\widetilde{u})} \geq 0$, and $E\left\{\varepsilon^{(u)}\right\} \geq E\left\{\varepsilon^{(\widetilde{u})}\right\}$, it is highly probable that the value of $u$ which minimizes $\varepsilon^{(u)}$ corresponds to $\tilde{u}$. From (37), it follows that

$$
\widehat{\mathbf{Y}}_{p}^{(u)}-\mathbf{Y}_{p}=\left(\operatorname{diag}\left\{\boldsymbol{\Phi}_{p}^{(u)}\right\} \boldsymbol{\Psi}_{p} \operatorname{diag}\left\{\boldsymbol{\Phi}_{p}^{(u)}\right\}^{H}-\mathbf{I}_{K_{p}}\right) \mathbf{Y}_{p} .
$$

From (38), (40), and (44), it is easy to know that the SI $\widetilde{u}$ can be estimated at the receiver by minimizing the candidate metric $\varepsilon^{(u)}=\left\|\Gamma_{p}^{(u)} \mathbf{Y}_{p}\right\|^{2}$ as

$$
\widehat{u}=\arg \min _{u \in\{0,1, \cdots, U-1\}}\left\|\boldsymbol{\Gamma}_{p}^{(u)} \mathbf{Y}_{p}\right\|^{2},
$$

where

$$
\begin{aligned}
\boldsymbol{\Gamma}_{p}^{(u)}= & \operatorname{diag}\left\{\boldsymbol{\Phi}_{p}^{(u)}\right\} \operatorname{diag}\left\{\mathbf{X}_{p}\right\} \mathbf{D}_{p}\left(\mathbf{D}_{p}^{H} \mathbf{D}_{p}\right)^{-1} \mathbf{D}_{p}^{H} \\
& \times \operatorname{diag}\left\{\mathbf{X}_{p}\right\} \operatorname{diag}\left\{\boldsymbol{\Phi}_{p}^{(u)}\right\}^{H}-\mathbf{I}_{K_{p}} .
\end{aligned}
$$

To reduce computational complexity, the $U$ matrices, $\left\{\boldsymbol{\Gamma}_{p}^{(u)}\right\}_{u=0}^{U-1}$, can be computed off-line and saved in memory for later use. Then, the SI estimation based on (45) requires $U K_{p}^{2}$ complex multiplications $(\mathrm{CMs}), U K_{p}\left(K_{p}-1\right)$ complex additions (CAs), $U K_{p}$ operations of $|\cdot|^{2}$, and $U\left(K_{p}-1\right)$ real additions (RAs). Since one $\mathrm{CM}$ is translated into 4 real multiplications (RMs) and 2 RAs, one CA is translated into 2 RAs, and one operation of $|\cdot|^{2}$ is translated into 2 RMs and 1 RA [22], the computational complexity of the SI estimation based on (45) corresponds to $\left(4 K_{p}^{2}+2 K_{p}\right) U$ RMs and $\left(4 K_{p}^{2}-1\right) U$ RAs. Once $\tilde{u}$ is estimated, the $M$-ary QAM symbols can be estimated by the symbol-based ML detection, which requires additional computation of $(2 M+4) K_{d}$ RMs and $(3 M+2) K_{d}$ RAs.

\section{Numerical Results}

For simulation, the system parameters are chosen as $K=512$, $K_{d}=480, K_{p}=32$, and $L=16$. In order to accommodate the nonlinear amplification characteristics, Rapp's solid-state power amplifier (SSPA) model [27] is adopted with smoothness factor 3 and input back-off factor $6 \mathrm{~dB}$. In this section, the proposed BSLM scheme and four traditional BSLM schemes are compared by simulation:

(i) PROPOSED: the BSLM scheme presented in the previous section.

(ii) BSLM-I: the BSLM scheme in [19], which applies the simplified ML decoding.

(iii) BSLM-II: the BSLM scheme in [20], which applies partition and phase offset techniques with its partition number given by $U / 2$.

(iv) BSLM-III: the BSLM scheme in [21], which applies the power-extended scrambling.

(v) BSLM-IV: the BSLM scheme in [22], which uses the autocorrelation between the CFR coefficients over the adjacent pilot subcarriers.

Figure 1 shows the SIER results of the five BSLM schemes in two cases of $\sigma_{T} / T_{S}=2$ and $\sigma_{T} / T_{S}=9$ when $U=8$. It can be seen that PROPOSED yields lower SIERs than other BSLM schemes in both the cases of $\sigma_{T} / T_{S}=2$ and $\sigma_{T} / T_{S}=9$. In the case of $\sigma_{T} / T_{S}=2$, BSLM-IV yields SIERs comparable to those of PROPOSED. However, in the case of $\sigma_{T} / T_{S}=9$, BSLMIV degrades severely yielding the worst SIER performance. It is because a large valued $\sigma_{T} / T_{S}$ incurs a greater number of effective channel taps leading to more severely fluctuating CFT amplitudes and more weakened CFR autocorrelation. Since the SI estimation in BSLM-IV is based on the high CFR autocorrelation assumption, the SIER performance of BSLM-IV degrades as $\sigma_{T} / T_{S}$ increases. Contrary to BSLMIV, PROPOSED shows good SIER performance regardless of $\sigma_{T} / T_{S}$.

In Figure 2, the complementary cumulative distribution functions (CCDFs) of the five BSLM schemes are plotted for various values of $U$ when the transmitted symbols are chosen from the 16QAM constellation. The CCDF is defined as

$$
P_{\mathrm{CCDF}}=\operatorname{Pr}\left(\mathrm{PAPR}>\mathrm{PAPR}_{0}\right),
$$

which means the probability that the PAPR is greater than a given quantity, $\mathrm{PAPR}_{0}$. For referential purpose, NONSLM is included in the figure, which denotes the performance of the original OFDM that does not apply any SLM scheme. From the figure, it can be seen that all the BSLM schemes reduce the PAPR substantially when compared with NONSLM. Note that the five BSLM schemes show almost identical PAPR reduction. The amounts of PAPR reduction by the five BSLM schemes tend to increase as the number of scrambling sequences increases.

Figure 3 shows the BER results of PROPOSED for various values of $U$ when $\sigma_{T} / T_{S}=2$ and the transmitted symbols are chosen from the 16QAM constellation. For referential purpose, NONSLM is included in the figure, which denotes 


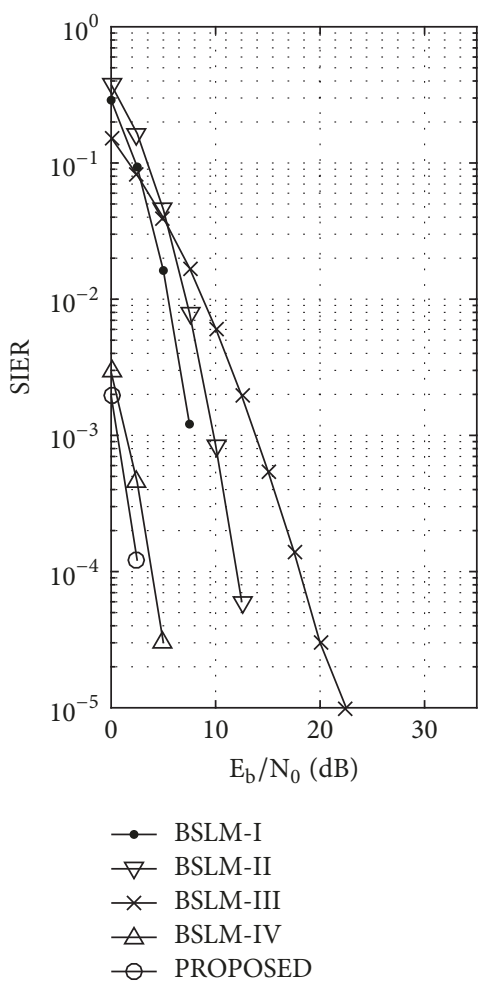

(a)

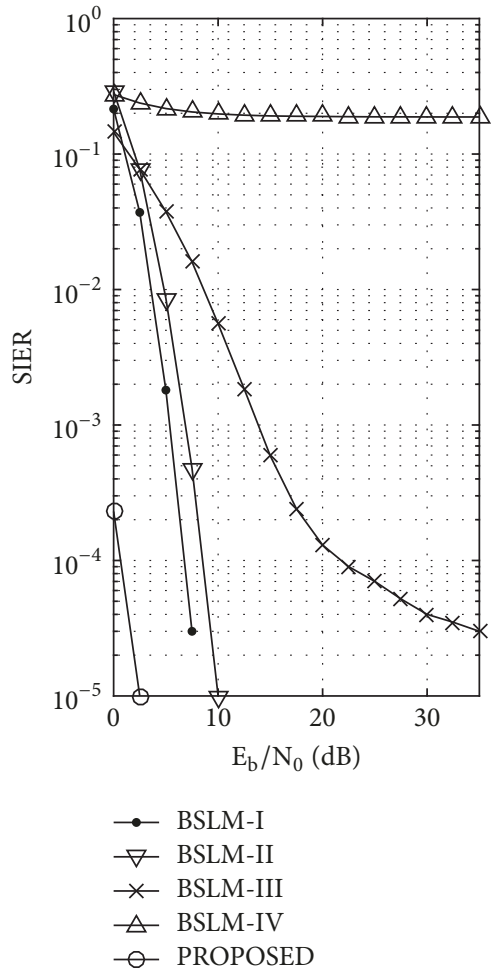

(b)

FIGURE 1: Comparison of the SIER results in two cases of (a) $\sigma_{T} / T_{S}=2$ and (b) $\sigma_{T} / T_{S}=9$ when $K=512, K_{d}=480, K_{p}=32$, and $U=8$. The transmitted symbols are chosen from the 16QAM constellation.

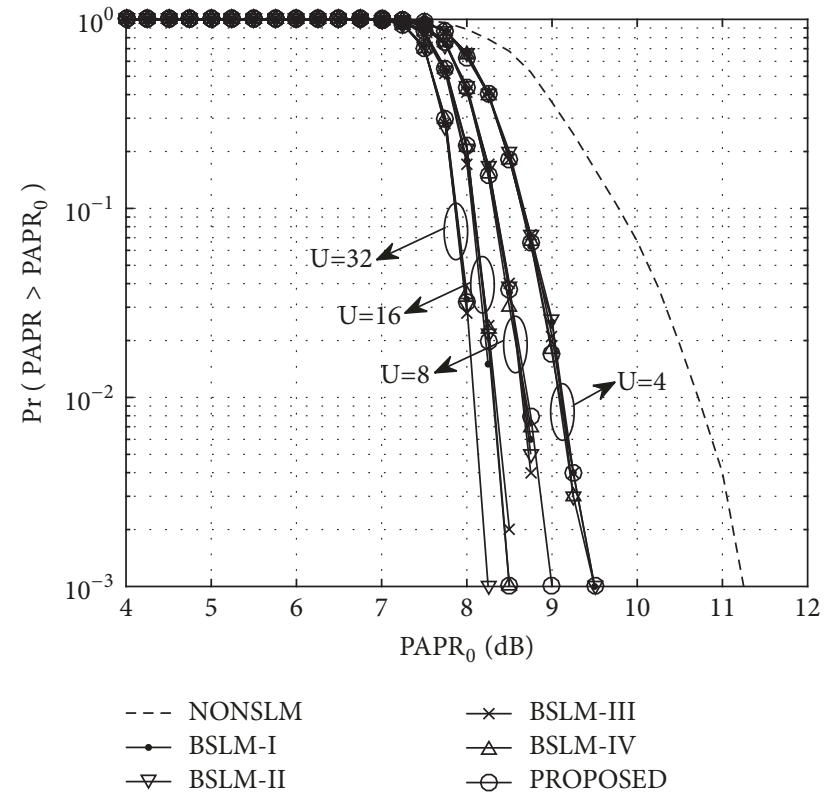

FIGURE 2: Comparison of the CCDF results for various values of $U$ when $K=512, K_{d}=480, K_{p}=32, \sigma_{T} / T_{S}=2$, and the transmitted symbols are chosen from the 16QAM constellation.

the performance of the original OFDM that does not apply any SLM scheme. It can be seen that PROPOSED improves the BER performance substantially for all the values of $U$

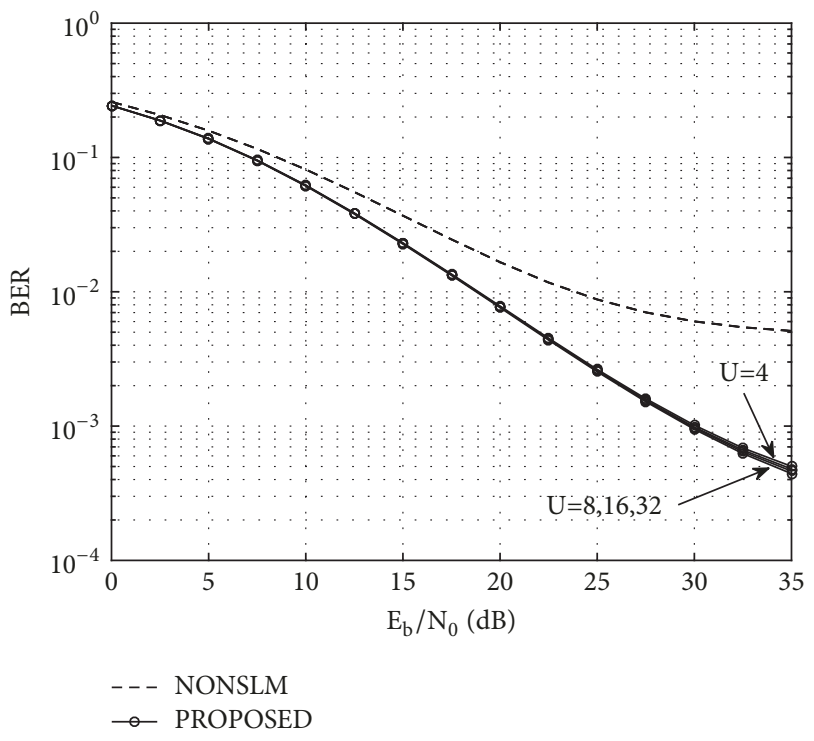

FIGURE 3: Comparison of the BER results of PROPOSED for various values of $U$ when $K=512, K_{d}=480, K_{p}=32, \sigma_{T} / T_{S}=2$, and the transmitted symbols are chosen from the 16QAM constellation.

when compared to NONSLM. The BER improvement by PROPOSED with increasing $U$ appears to reach saturation when $U=8$. It implies that increasing the number of 


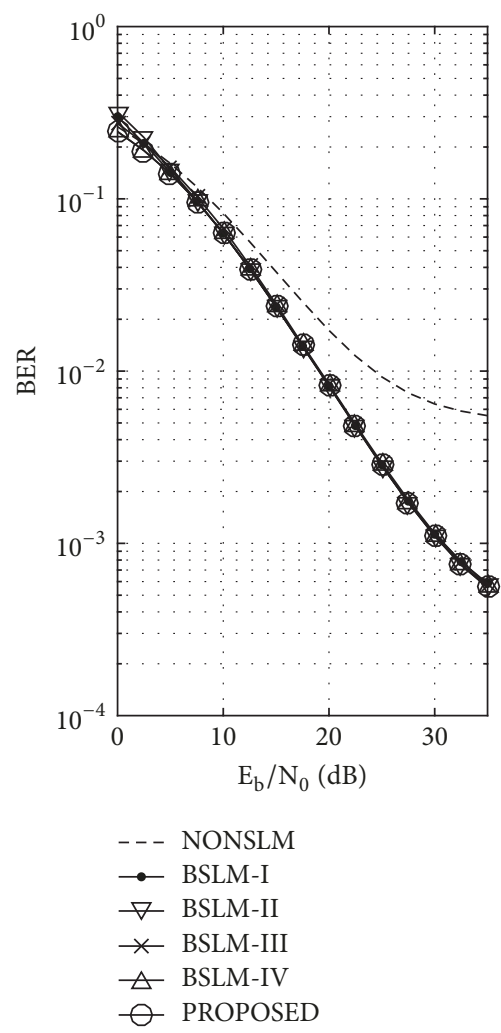

(a)

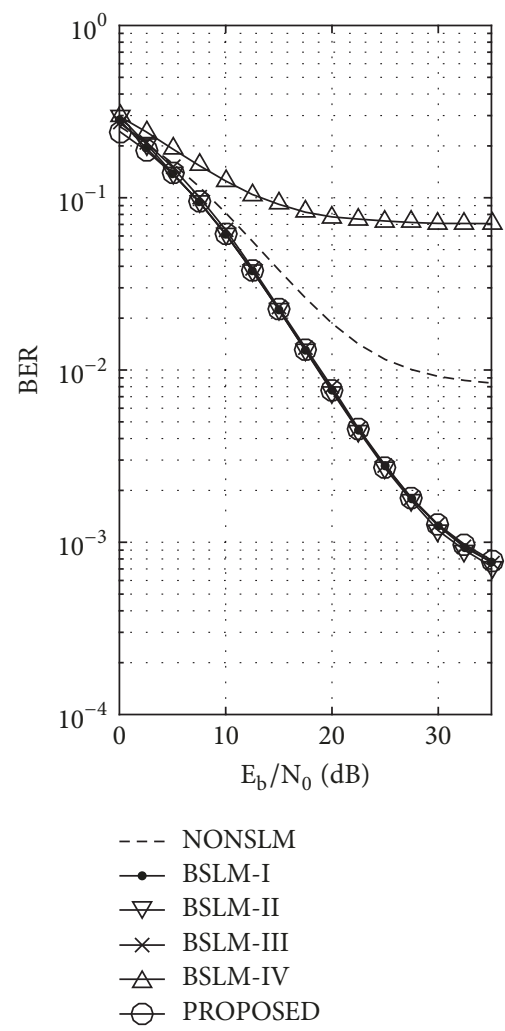

(b)

Figure 4: Comparison of the BER results in two cases of (a) $\sigma_{T} / T_{S}=2$ and (b) $\sigma_{T} / T_{S}=9$ when $K=512, K_{d}=480, K_{p}=32, U=8$, and the transmitted symbols are chosen from the 16QAM constellation.

the scrambling sequences over 8 brings only trivial BER improvement while it further complicates SI estimation. Therefore, for the remaining part of this section, $U=8$ is chosen in simulation.

Figure 4 shows the BER results of the five BSLM schemes in two cases of $\sigma_{T} / T_{S}=2$ and $\sigma_{T} / T_{S}=9$ when the transmitted symbols are chosen from the 16QAM constellation. In the case of $\sigma_{T} / T_{S}=2$, it can be seen that PROPOSED and BSLMIV yield lower BERs than other BSLM schemes. In the case of $\sigma_{T} / T_{S}=9$, BSLM-IV yields severely degraded BERs even worse than the BERs of NONSLM. On the contrary, in the case of $\sigma_{T} / T_{S}=9$, PROPOSED outperforms all the other BSLM schemes. In both the cases of $\sigma_{T} / T_{S}=2$ and $\sigma_{T} / T_{S}=9$, BSLM-I shows BERs comparable to those of PROPOSED at a high SNR. Since PROPOSED has lower implementation complexity than BSLM-I, PROPOSED can be regarded as more efficient than BSLM-I. BSLM-II shows slightly worse BER performance than other BSLM schemes at a low SNR.

Figure 5 shows the BER results of the five BSLM schemes under the same simulation conditions as in Figure 4 except that the transmitted symbols are chosen from the 64QAM constellation. By comparing Figure 4 and Figure 5, it can be seen that BSLM-I, BSLM-II, BSLM-IV, and PROPOSED show the same BER trends in both figures. However, BSLM-III degrades severely as the symbol constellation size increases. In addition, it can be seen that BSLM-III degrades further as $\sigma_{T} / T_{S}$ increases. It is because a large symbol constellation size or a large valued $\sigma_{T} / T_{S}$ incurs a large variation of the received signal power, which makes it difficult for BSLMIII to estimate the SI correctly by using the locations of the power-extended scrambling symbols.

In Figure 6, the implementation complexities of the BSLM schemes are compared. As in [20], the detection complexity reduction ratio (DCRR) is defined as

$$
\begin{aligned}
& \text { DCRR } \\
& =1-\frac{\text { Complexity of the proposed BSLM }}{\text { Complexity of the conventional BSLM }}
\end{aligned}
$$

Note that a positive DCRR value indicates that PROPOSED has lower implementation complexity than the conventional BSLM scheme being considered. In computing the complexity, both the complexity of SI estimation and that of symbol detection are considered together. The RM-based and RA-based DCRRs of BSLM-I, BSLM-II, BSLM-III, and BSLM-IV are computed for various QAM constellation sizes by referring to the complexity analysis in $[20,22]$ and plotted in Figure 6. It can be seen that the RM-based and RA-based DCRRs of BSLM-I, BSLM-II, and BSLM-III are positive for all the QAM constellation sizes. In addition, the RM-based and RA-based DCRRs of BSLM-IV are positive when $M>4$. This implies that PROPOSED has lower implementation complexity than the four traditional BSLM 


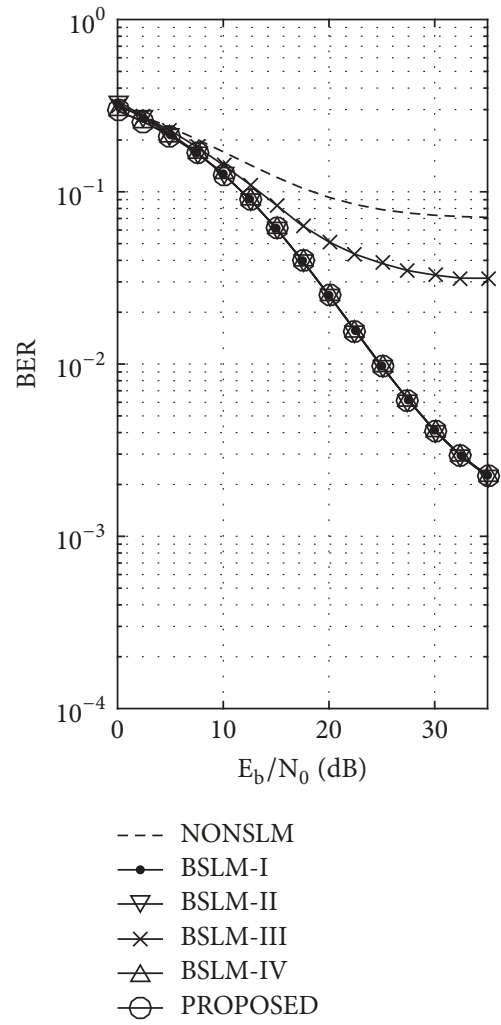

(a)

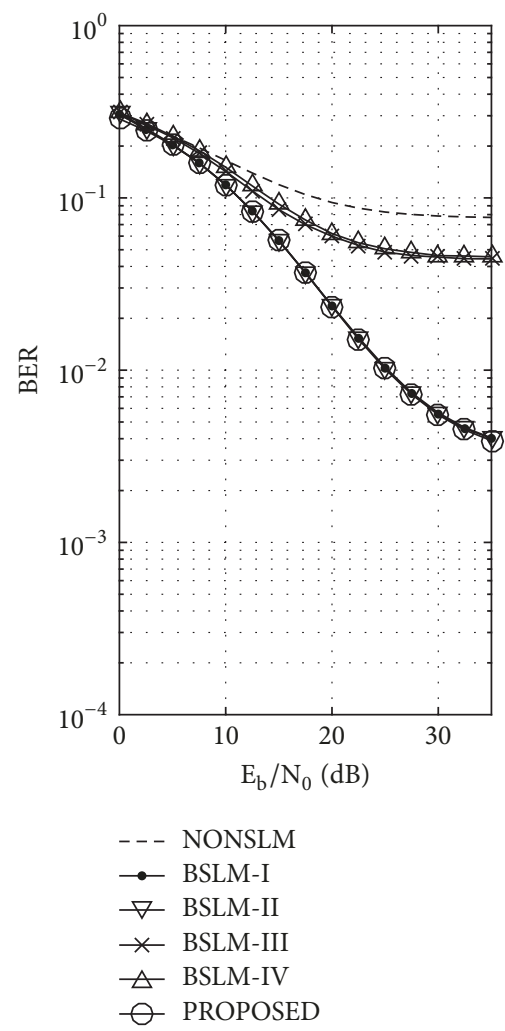

(b)

Figure 5: Comparison of the BER results in two cases of (a) $\sigma_{T} / T_{S}=2$, and (b) $\sigma_{T} / T_{S}=9$ when $K=512, K_{d}=480, K_{p}=32, U=8$, and the transmitted symbols are chosen from the 64QAM constellation.

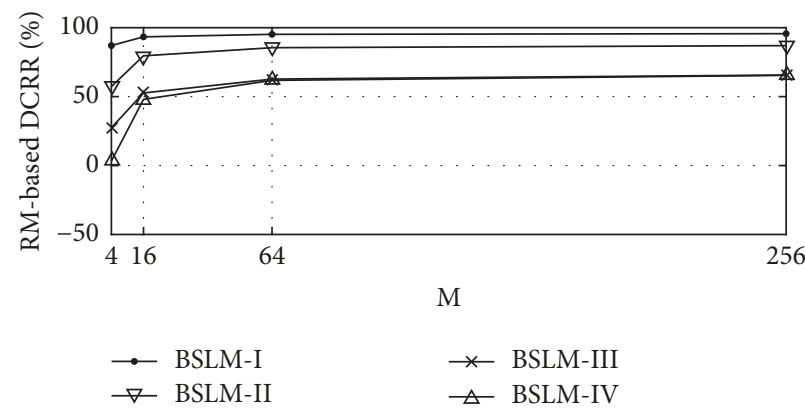

(a)

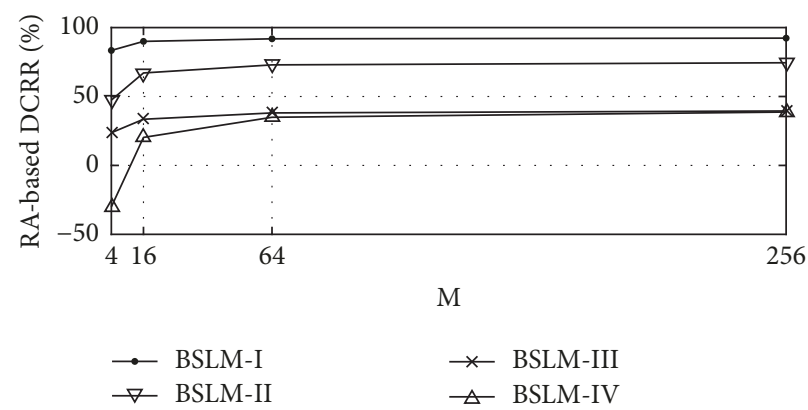

(b)

FIGURE 6: Comparison of the DCRR results in terms of the QAM constellation size, $M$, which were computed by using (a) RMs and (b) RAs, respectively, when $K=512, K_{d}=480, K_{p}=32$, and $U=8$.

schemes. The RM-based and RA-based DCRRs of BSLMI, BSLM-II, BSLM-III, and BSLM-IV tend to increase as the QAM constellation size increases. This implies that the amount of complexity reduction by PROPOSED increases as the symbol constellation size increases.

\section{Conclusion}

A BSLM scheme that applied a new SI estimation approach based on the pilot signal was proposed. The proposed BSLM scheme estimated the SI by selecting the minimum of the candidate metrics that were computed with the received pilot signal, the pilot symbol sequence, and the scrambling sequences. It was shown by simulation that the proposed BSLM scheme yielded better SIER and BER performance and lower implementation complexity than four traditional BSLM schemes.

\section{Conflicts of Interest}

The authors declare that there are no conflicts of interest regarding the publication of this paper. 


\section{Acknowledgments}

This paper was supported by Konkuk University in 2016.

\section{References}

[1] Y. Wu and W. Y. Zou, "Orthogonal frequency division multiplexing: A multi-carrier modulation scheme," IEEE Transactions on Consumer Electronics, vol. 41, no. 3, pp. 392-399, 1995.

[2] N. Bhushan, T. Ji, O. Koymen et al., "5G Air Interface System Design Principles," IEEE Wireless Communications Magazine, vol. 24 , no. 5 , pp. $6-8,2017$.

[3] J. Tellado, Multicarrier Modulation with Low PAR: Applications to DSL and Wireless, Kluwer, Norwell, Mass, USA, 2000.

[4] T. Jiang and Y. Wu, "An overview: peak-to-average power ratio reduction techniques for OFDM signals," IEEE Transactions on Broadcasting, vol. 54, no. 2, pp. 257-268, 2008.

[5] A. E. Jones, T. A. Wilkinson, and S. K. Barton, "Block coding scheme for reduction of peak to mean envelope power ratio of multicarrier transmission schemes," IEEE Electronics Letters, vol. 30, no. 25, pp. 2098-2099, 1994.

[6] J. Zakaria and M. F. M. Salleh, "PAPR reduction scheme: Wavelet packetbased PTS with embedded side information data scheme," IET Communications, vol. 11, no. 1, pp. 127-135, 2017.

[7] X. Li and L. J. Cimini Jr., "Effects of clipping and filtering on the performance of OFDM," IEEE Communications Letters, vol. 2, no. 5, pp. 131-133, 1998.

[8] J. Tellado, Peak to Average Power Ratio Reduction for Multicarrier Moulation [Ph.D. thesis], University of Stanford, Stanford, 1999.

[9] X. Wang, T. T. Tjhung, and C. S. Ng, "Reduction of peakto-average power ratio of OFDM system using a companding technique," IEEE Transactions on Broadcasting, vol. 45, no. 3, pp. 303-307, 1999.

[10] V. Tarokh and H. Jafarkhani, "On the computation and reduction of the peak-to-average power ratio in multicarrier communications," IEEE Transactions on Communications, vol. 48, no. 1, pp. 37-44, 2000.

[11] Y. Kou, W.-S. Lu, and A. Antoniou, "New peak-to-average power-ratio reduction algorithms for multicarrier communications," IEEE Transactions on Circuits and Systems I: Regular Papers, vol. 51, no. 9, pp. 1790-1800, 2004.

[12] S. H. Han and J. H. Lee, "PAPR reduction of OFDM signals using a reduced complexity PTS technique," IEEE Signal Processing Letters, vol. 11, no. 11, pp. 887-890, 2004.

[13] H. Kim, E. Hong, C. Ahn, and D. Har, "A pilot symbol pattern enabling data recovery without side information in PTS-based OFDM systems," IEEE Transactions on Broadcasting, vol. 57, no. 2, pp. 307-312, 2011.

[14] Y. Rahmatallah and S. Mohan, "Peak-to-average power ratio reduction in ofdm systems: a survey and taxonomy," IEEE Communications Surveys \& Tutorials, vol. 15, no. 4, pp. 15671592, 2013.

[15] X. Huang, G. Tan, Q. Xu, N. Xu, and S. Wang, "A kind of PAPR reduction method based on pruning WPM and PTS technology," Journal of Electronics (China), vol. 30, no. 3, pp. 261-267, 2013.

[16] L. Li, D. Qu, and T. Jiang, "Partition optimization in LDPCcoded OFDM systems with PTS PAPR reduction," IEEE Transactions on Vehicular Technology, vol. 63, no. 8, pp. 4108-4113, 2014.
[17] H.-S. Joo, K.-H. Kim, J.-S. No, and D.-J. Shin, "New PTS Schemes for PAPR Reduction of OFDM Signals Without Side Information," IEEE Transactions on Broadcasting, vol. 63, no. 3, pp. 562-570, 2017.

[18] R. W. Bäuml, R. F. H. Fischer, and J. B. Huber, "Reducing the peak-to-average power ratio of multicarrier modulation by selected mapping," IEEE Electronics Letters, vol. 32, no. 22, pp. 2056-2057, 1996

[19] A. D. S. Jayalath and C. Tellambura, "SLM and PTS peak-power reduction of OFDM signals without side information," IEEE Transactions on Wireless Communications, vol. 4, no. 5, pp. 2006-2013, 2005.

[20] H.-S. Joo, S.-J. Heo, H.-B. Jeon, J.-S. No, and D.-J. Shin, "A new blind SLM scheme with low decoding complexity for OFDM systems," IEEE Transactions on Broadcasting, vol. 58, no. 4, pp. 669-676, 2012.

[21] S. Y. Le Goff, S. S. Al-Samahi, B. K. Khoo, C. C. Tsimenidis, and B. S. Sharif, "Selected mapping without side information for PAPR reduction in OFDM," IEEE Transactions on Wireless Communications, vol. 8, no. 7, pp. 3320-3325, 2009.

[22] E. Hong, H. Kim, K. Yang, and D. S. Har, "Pilot-aided side information detection in SLM-based OFDM systems," IEEE Transactions on Wireless Communications, vol. 12, no. 7, pp. 3140-3147, 2013.

[23] T. Kitazawa and K. Ohuchi, "On blind estimation with reduced complexity in CSS-OFDM systems," in Proceedings of the 2016 IEEE International Symposium on Signal Processing and Information Technology, ISSPIT 2016, pp. 120-125, cyp, December 2016.

[24] B. Wang, Y. Wu, F. Han, Y.-H. Yang, and K. J. R. Liu, "Green wireless communications: A time-reversal paradigm," IEEE Journal on Selected Areas in Communications, vol. 29, no. 8, pp. 1698-1710, 2011.

[25] M. Emami, M. Vu, J. Hansen, A. J. Paulraj, and G. Papanicolaou, "Matched filtering with rate back-off for low complexity communications in very large delay spread channels," in Proceedings of the Conference Record of the Thirty-Eighth Asilomar Conference on Signals, Systems and Computers, pp. 218-222, usa, November 2004.

[26] M. Morelli and U. Mengali, "A comparison of pilot-aided channel estimation methods for OFDM systems," IEEE Transactions on Signal Processing, vol. 49, no. 12, pp. 3065-3073, 2001.

[27] C. Rapp, "Effects of HPA-nonlinearity on 4-DPSK OFDM Signal for a Digital Sound Broadcasting System," in Proceedings of 2 nd European Conference on Satellite Commununications, pp. 179-184, Liege, Belgium, 1991. 


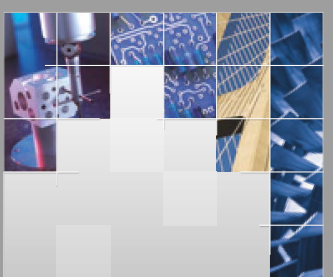

\section{Enfincering}
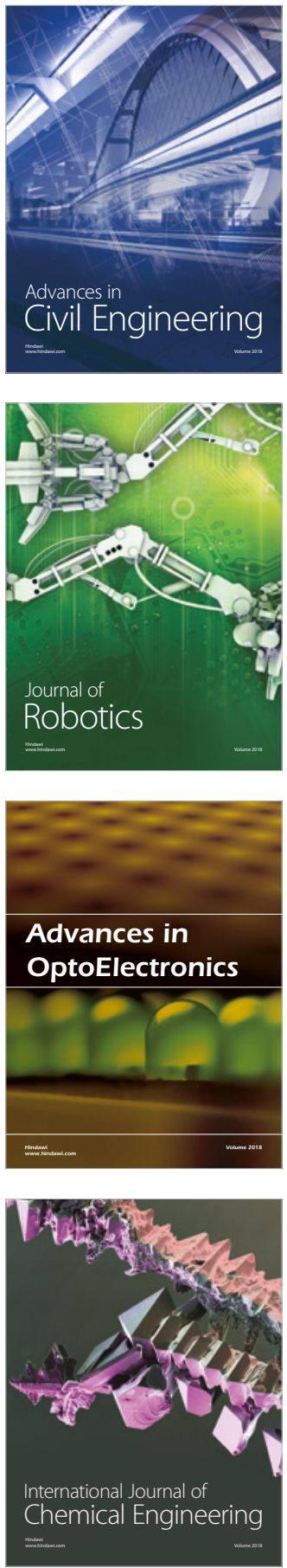

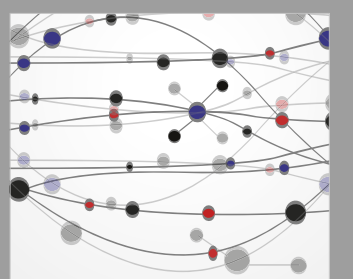

\section{Rotating \\ Machinery}

The Scientific World Journal

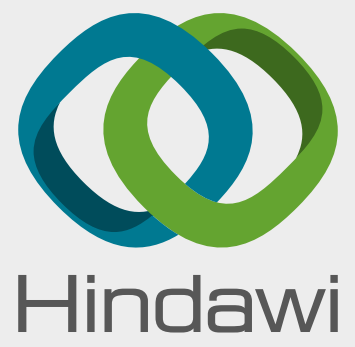

Submit your manuscripts at

www.hindawi.com
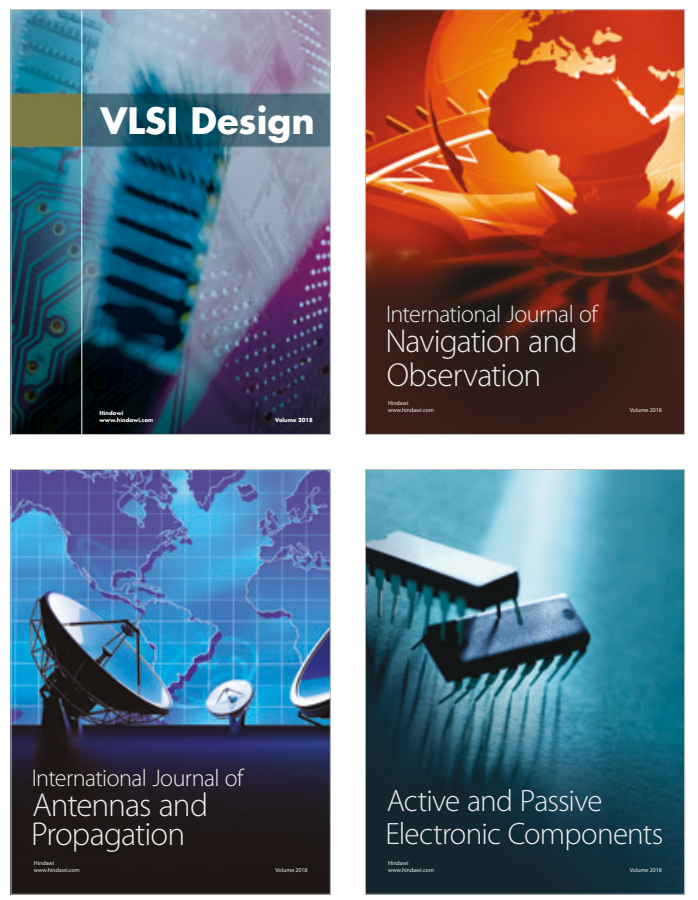
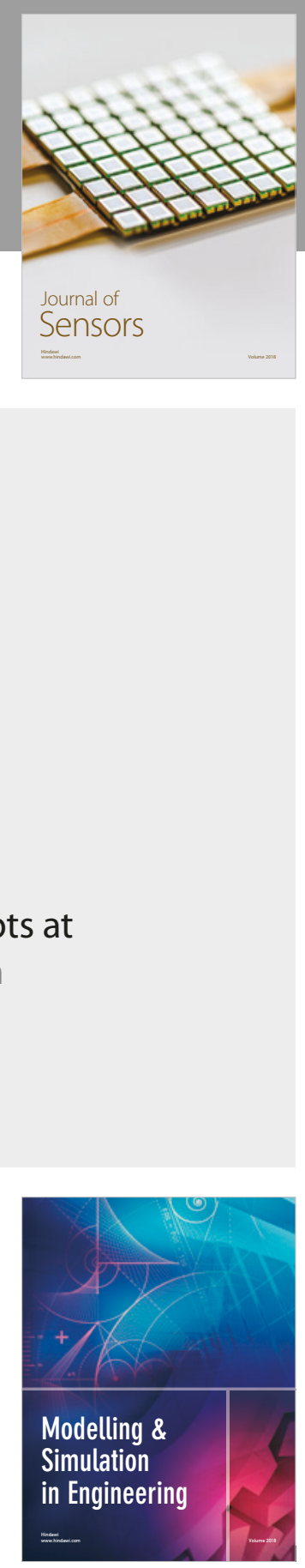

\section{Advances \\ Multimedia}
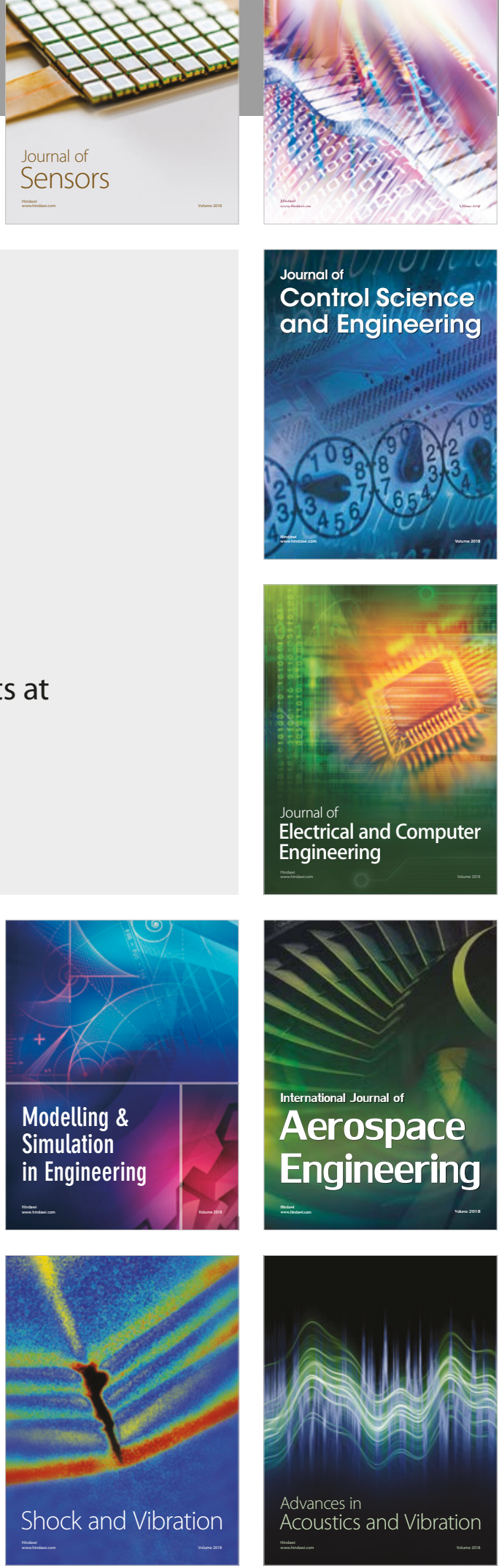